\title{
Molecular investigation of bacterial communities on inner and outer surfaces of peripheral venous catheters
}

Li Zhang $^{1 *}$, Mark Morrison ${ }^{2}$, Graeme R. Nimmo ${ }^{3}$, Kadaba S. Sriprakash ${ }^{4}$, Stanislas Mondot $^{2}$, John R Gowardman ${ }^{5}$, Narelle George ${ }^{3}$, Nicole Marsh ${ }^{1,6}$, Claire M. Rickard ${ }^{1,5,6}$

${ }^{1}$ Research Centre for Clinical and Community Practice Innovation, Griffith University, Brisbane, Australia;

${ }^{2}$ CSIRO Livestock Industries, Queensland Bioscience Precinct, Brisbane, Australia;

${ }^{3}$ Division of Microbiology, Pathology Queensland Central, Brisbane, Australia;

${ }^{4}$ Bacterial Pathogenesis Laboratory, Queensland Institute of Medical Research, Brisbane, Australia;

${ }^{5}$ Department of Intensive Care Medicine, Royal Brisbane and Women's Hospital, Brisbane, Australia;

${ }^{6}$ Centre for Clinical Nursing, Royal Brisbane and Women's Hospital, Herston, Brisbane, Australia

*Correspondence: Li Zhang, N48 Nathan Campus, Griffith University, 170 Kessels Road, Nathan QLD 4111, Australia. Tel: +61 7 37357272; Fax: +61 7 3735 3560; Email:li.zhang@griffith.edu.au.

Running title: Bacterial diversity on peripheral venous catheters. 


\begin{abstract}
Peripheral venous catheters (PVCs) are some of the most widely used medical devices in hospitals worldwide. PVC-related infections increased morbidity and treatment costs. Inner surfaces of PVCs are rarely examined for population structure of bacteria as it is generally believed that bacteria at this niche are similar to those on the external surface of PVC. We primarily test this hypothesis and also study the effect of antibiotic treatment on bacterial communities from PVC surfaces. Inner and outer surfaces of PVCs from 15 patients were examined by 454 GS FLX Titanium 16S rRNA sequencing and the rell-plate' culture method. None of the PVCs were colonized according to the culture method and none of patients had a bacteraemia. From a total of 127,536 high quality sequence reads, 14 bacterial phyla and 268 diverse bacterial genera were detected. The number of Operational Taxonomic Units for each sample was in the range of 86-157 even though $60 \%$ of patients had received antibiotic treatment. Stenotrophomonas maltophilia was the predominant bacterial species in all examined PVC samples. There were noticeable but not statistically significant differences between the inner and outer surfaces of PVCs in terms of the distribution of the taxonomic groups. In addition, bacterial communities on PVCs from antibiotic treated patients were significantly different from untreated patients. In conclusion, surfaces of PVCs display complex bacterial communities although their significance is yet to be determined, these findings alter our perception of PVC-related infections.
\end{abstract}

Key words: Peripheral venous catheters; Catheter-related infections; Bacterial community; 16S rRNA gene sequences 


\section{Introduction}

2 Peripheral venous catheters (PVCs) are vital for the administration of fluids and medications

3 and for multiple blood access. In the USA, it is estimated that 25 million PVCs are inserted

4 each year, accounting for $90 \%$ of all venous catheters [1]. PVC-associated infection is

5 considered to be implicated in $5 \%$ of cases of nosocomial bacteraemia which has a

6 prevalence of 670 per 100,000 patients with PVCs [2]. Although PVC-related infections may

7 not result in increased mortality, they contribute considerably to the burden of the health-care

8 system by increasing morbidity and treatment costs $[1,3,4]$.

9 Intravenous catheter insertion provides a portal of entry for bacteria to cross from an unsterile external environment to the normally sterile blood [5]. The precursor to bloodstream infection (BSI) is usually device colonization; bacteria found adhering to the extra or intra luminal surfaces of catheters are the principal source and cause of BSIs [6,7]. Postattachment, many microbial communities form biofilms [8,9]. Because of increased resistance to antibiotics, the bacteria within biofilms are difficult to treat effectively [10].

Detection and identification of the bacteria found on intravascular catheters, including PVCs, has relied on culture-dependent techniques such as the 'roll-plate' [11]. This method involves culturing the external surface of the catheter tip by rolling a segment $(2-3 \mathrm{~cm})$ of the intravascular catheter four times over the surface of a blood agar plate with sterile forceps. However, the 'roll-plate' technique fails to detect bacteria on the inner surface of catheters and does not identify fastidious bacterial species. Moreover, bacteria identified from the positive cultures are rarely found to be associated with BSI [12-14]. As for the inner surfaces of short-term catheters, they are generally not examined for colonization as it is presumed that bacteria first colonize the outer surface before colonizing the inner surface, and that the composition of bacterial population at these two surfaces are similar. However, the 
1 availability of nutrition, temperature, oxygen tension, exposure to antibiotics and infusion

2 fluids, and fluid dynamics are factors that could contribute to differences in the micro-

3 environment at the inner and outer surfaces of PVCs. This may reflect in changes in bacterial

4 composition at both PVC surfaces. The aim of this study was to provide data to test this

5 hypothesis and to examine the effect of antibiotic treatment on microbial communities at

$6 \quad$ PVC surfaces.

\section{$7 \quad$ Materials and methods}

\section{$8 \quad$ Hospital setting and study population}

9 After obtaining institutional ethics approval and patients' informed consent, the study was 10 conducted from April 2008 to October 2009 at three teaching hospitals in Queensland, 11 Australia. PVCs (Insyte ${ }^{\mathrm{TM}}$ Autoguard $^{\mathrm{TM}}$ BD Medical, Franklin Lakes) were inserted by hospital nurses or doctors and cared for in accordance with usual hospital practice. Skin was decontaminated with $2 \%$ chlorhexidine gluconate in $70 \%$ ethanol before insertion of PVC. Transparent, semi-permeable dressings at the insertion site $\left(\right.$ Tegaderm $^{\mathrm{TM}} 1624 \mathrm{~W}, 3 \mathrm{M}$, St Paul) were maintained by nurses according to hospital protocol.

When the PVC was no longer required, the nursing staff removed the PVC after treating the insertion site with $2 \%$ chlorhexidine in $70 \%$ ethanol. Fifteen PVC samples were taken by qualified registered nurses with experience in preparation of specimens for culture. The distal 2-3 $\mathrm{cm}$ of the tip was cut using sterile scissors and deposited in a sterile container. All PVC tips were handled under aseptic conditions and immediately transported to the laboratory for examination, where they were cultured by the semi-quantitative method [11].

\section{Culture method}

This method is based on rolling a segment, usually the tip, of the removed PVCs back and forth on 5\% sheep blood agar plates (Oxoid, Australia). The plates were incubated at $37^{\circ} \mathrm{C}$ 
1 under aerobic conditions for 2-4 days. Microorganisms were then isolated and identified

2 according to standard microbiology protocols. The PVC tip was considered colonized if the

3 plate grew $\geq 15$ colony forming units (cfu).

\section{Molecular techniques}

5 In order to separate the bacterial communities of inner and outer PVC surfaces, $1 \mathrm{~cm}$ at both

6 ends of PVC tips were carefully heat-sealed. PVC tips were placed in $1.5 \mathrm{ml}$ microcentrifuge

7 tubes and gently sonicated to release the bacteria from the surfaces of the PVCs. Lysis buffer 8 containing $20 \mathrm{mg} / \mathrm{ml}$ lysozyme, $20 \mathrm{mM}$ Tris- $\mathrm{HCl}$ (pH 8.0), $2 \mathrm{mM}$ EDTA, $1.2 \%$ Triton was 9 added and incubated at $37{ }^{\circ} \mathrm{C}$ overnight. After that, PVC tips were taken out and bacterial DNA from outer surfaces of PVCs was collected. DNA extraction was continued using QIAamp DNA mini kit (Qiagen, Australia) according to the manufacturer's instructions.

In order to extract bacterial DNA from the inner surface, the external surface of the PVC section was washed and disinfected with ethanol, both ends of PVC tips were cut and $200 \mu 1$ of lysis buffer passed through. The PVC fragment was incubated at $37{ }^{\circ} \mathrm{C}$ overnight. Bacterial DNA from inner surfaces was collected and extracted using QIAamp DNA mini kit (QIAGEN, Australia) according to the manufacturer's instructions. A new PVC was suspended in lysis buffer and DNA was extracted as above for a negative control. 16S rRNA genes were amplified from purified genomic DNA using the primers $\mathrm{F}$ (5' AGA GTT TGA TCC TGG CTC AG 3') and R (5' GAG TTT GAT CCT GGC TCA G 3') which would cover variable regions (V1, V2 and V3). Thirty different self correction barcodes were designed and added to primers. For each $25 \mu 1$ reaction, conditions were as follows: $3 \mu 1$ of DNA template (concentration ranged from neat to $1: 10^{3}$ ), $2.5 \mu$ of $10 \times$ reaction buffer containing $20 \mathrm{mM} \mathrm{MgCl} 2,2 \mu \mathrm{l}$ of $25 \mathrm{mM}$ dNTPs, $1 \mu \mathrm{l}$ of each primer $(10 \mu \mathrm{M}), 0.1 \mathrm{U}$ of Taq DNA polymerase (Qiagen, Australia), $5 \mu \mathrm{l}$ of $5 \times \mathrm{BSA}$ and $10.4 \mu \mathrm{l}$ of sterile deionized water. Each 
1 PCR run contained a negative control (sterile deionized water instead of template DNA) and

2 a positive control (E. coli instead of template DNA). For each DNA sample, three replicate

3 PCRs were performed.

4 PCR products were cleaned using Qiaquick PCR Purification kit (Qiagen, Australia). These

5 were sequenced unidirectionally in the reverse direction by means of the Genome Sequencer

$6 \quad$ FLX (GS-FLX) system (Roche, Australia) at 454 Life Sciences.

\section{Informatics analysis}

8 Pyrosequence reads were analysed using QIIME package (Quantitative Insights Into

9 Microbial Ecology) as follows [15]. Briefly, bar coded 16S rRNA gene sequences were inputed into QIIME, then sequences were classified using the Ribosomal Database Project classifier, and sequences were aligned and chimera checked using Pynast and ChimeraSlayer. UniFrac distances were calculated, and data generated for summarizing the proportions of taxonomy and Principle Coordinates Analysis (PCoA) plots. All sequences were assigned to phylotypes (Operational Taxonomic Units, OTUs) at 97\% sequence identity thresholds and $80 \%$ confidence was used in the QIIME-based wrapper of the RDP classifier program against the RDP core set. The Chao1 microbial richness estimate and overall community diversity (Shannon-Weaver index) were computed from the OTU data. Rarefaction curves were generated by plotting the number of observed genera versus the number of sequences sampled. A heatmap was generated on the basis of the relative abundance of OTUs using $\mathrm{R}$ version 2.15 (The R Project for Statistical Computing). PCoA was carried out based on thetasimilarities of the relative abundance of the OTU in each sample. The top bacterial OTUs were selected after ranking their separability measured as the variance between classes using principal component analysis with respect to instrumental variables in $\mathrm{R}$ version 2.15 [16]. 
1 bacterial groups were shown in box plots and two tailed $t$-Test was used to evaluate the

2 difference between PVC groups. The Monte Carlo permutation test was used to test the statistical significance of the relationships bacterial communities on PVCs between patients

$4 \quad$ with antibiotics and without antibiotics.

\section{$5 \quad$ Results and Discussion}

\section{Sample characteristics}

7 The 15 patients of whom $60 \%$ were females had a median age of 58 years. All PVCs were 8 short-term and duration of PVC left in situ varied from 2-4 days. Approximately $80 \%$ of 9 PVCs were in the forearm and $60 \%$ of patients were receiving systemic antibiotic therapy during the observation period. No local signs of inflammation were noticed. No patients were suspected or diagnosed with PVC-related BSI. According to the 'roll-plate' culture results, none of PVCs were colonized as no bacteria were grown on Columbia blood agar plates. In this study bacterial DNA was extracted from all PVC samples although they were "non-colonized" as defined by the 'roll-plate' method. These DNA samples were amplified and used for constructing pyrosequencing libraries. No bacterial DNA was detected and amplified from negative control PVCs. These control results indicate that the contribution to the bacterial community from manufacture of PVCs, DNA extraction procedures and PCR reagents is negligible.

\section{Features of the bacterial community on PVC}

Sequence lengths outside bounds of 150 and 600, ambiguous bases exceeding limits, missing or mean quality score below a minimum of 25 , maximum homopolymer run exceeding a limit of 6, and mismatches in primers exceeding limits of 1 were discarded. Of the 16S rRNA gene sequences generated, 127,536 high-quality sequence reads from 30 samples (15 outerand inner- surfaces of PVCs) passed the set threshold, and were used for further analysis. 
1 Chimera checks showed that all sequences were unlikely to be chimeric. The average number

2 of sequences per sample was 4251 (range 1413-10957) and average base-pair in length was

3496 (range 150-599).

4 Overall, microbial communities on PVCs had a high diversity and complex community

5 structure. 16S rRNA gene sequences were assigned to fourteen different bacterial phyla:

6 Acidobacteria, Actinobacteria, Bacteroidetes, Chloroflexi, Cyanobacteria, Deinococcus-

7 Thermus, Firmicutes, Fusobacteria, Gemmatimonadetes, Planctomycetes, Proteobacteria,

8 TM7, Thermotogae and unclassified bacteria (Fig. 1a). The predominant phylum was

9 Proteobacteria, including Alphaproteobacteria (4\% of 16S rRNA reads), Betaproteobacteria

$10(8.4 \%)$ and Gammaproteobacteria (68\%). Four phyla (Actinobacteria, Bacteroidetes,

11 Firmicutes and Proteobacteria) represented, on average 98\% of individual bacterial 12 communities on PVC surfaces. In all, 140 OTUs were from unclassified bacteria. All 13 examined sequences were assigned to 1026 OTUs (predominant OTUs were shown in Fig. 2).

14 A high number of OTUs was observed for each sample, ranging from 86 to 157. Sample richness was between 119 and 268, and the overall diversity (Shannon-Weaver index) was in the range of 2.24 and 3.64 .

Bacterial diversity was very complex when examined using pyrosequencing analysis.

The limitations of culture methods to determine bacterial communities from environmental and clinical samples have been reported previously [17]. Several possible explanations for the discordant results from the two methods included: 1) antibiotic usage in patients which are common for acute hospital patients [18-21]; 2) sonication as part of the protocol in molecular methods may help detachment of bacteria in biofilms; 3) some bacteria are more easily cultured than others; 4) DNA based molecular methods might detect DNA from dead bacteria. Overall, the molecular methods provide much greater sensitivity than culture methods for this work. 


\section{Comparisons between bacterial communities on inner and outer surfaces of PVCs}

2 Difference in Shannon-Weaver diversity indics between bacterial communities on inner and 3 outer surfaces of PVCs was not significant, with $p=0.365\left(H_{\text {Inner surface }}=3.05 \pm 0.35, H_{\text {Outer }}\right.$ 4 surface $=2.95 \pm 0.22$ ). Similarly, results from PCoA suggested that bacterial communities on 5 inner and outer surfaces of PVCs had no statistically significant difference, as they did not

6 separate. There were noticeable differences between the inner and outer surfaces of PVCs in

7 terms of the distribution of the taxonomic groups. For example, PVC inner surfaces had higher abundance of Firmicutes, Gemmatimonadetes, Planctomycetes and TM7 than the outer surfaces (Fig. 1b). More Acidobacteria, Actinobacteria, Bacteroidetes, Cyanobacteria and Proteobacteria were detected on the outer surfaces of PVCs than on the inner surfaces. Chloroflexi and Deinococcus-Thermus were only present on the inner surfaces, and conversely, Fusobacteria and Thermotogae were only present on the outer surfaces of PVCs.

It is unknown whether those low abundance bacteria play a significant role in PVC-related infections or not and future research $\underline{\text { is }}$ required to verify this. Although these results indicate variations in bacterial diversity, they are at very low abundance. No significant difference was found between the two groups.

\section{Effect of antibiotic treatment on bacterial communities of PVCs}

There were 12 PVC samples (inner and outer surfaces of PVCs) from six patients without antibiotic treatment. We compared bacterial communities on these to the 18 PVC samples from nine patients treated with intravenous antibiotics to those from untreated patients. Results from PCoA (Fig. 3), Shannon-Weaver diversity index $\left(H_{\text {Antibiotic }}=2.85 \pm 0.30\right.$, $H_{\text {Noantibiotic }}=3.13 \pm 0.21 ; p=0.005$ ) and Monte Carlo permutation test (Fig. 4) showed significantly different bacterial communities on PVCs from patients that were untreated and treated with antibiotics in the two weeks prior to PVC collection. The analysis found that 
1 PVCs from patients without antibiotic use were associated with a significantly higher

2 abundance of Thermomonas fusca, Flavobacteriaceae, Stenotrophomonas maltophilia,

3 Brevibacterium sp., Chryseobacterium sp., Streptococcus suis and Leucobacer sp.

4 Stenotrophomonas were the predominant bacteria on all examined PVCs (Fig. 5). However,

5 Stenotrophomonas abundance in patients with antibiotic treatment was dramatically

6 decreased compared with patients who were not treated with antibiotics and it was shown

7 in Fig. 5. PVCs from patients with antibiotic treatment had a significantly higher abundance

8 of Phyllobacterium sp., Propionibacterium acnes, Acidovorax sp. and Escherichia coli (Fig.

9 5). Results from this study show that antibiotic therapy reduces bacterial diversity and alters

10 the structure of bacterial communities-reducing some bacteria while selecting for others.

11 More attention should be paid on bacterial communities of PVCs from patients with antibiotic treatment since they have great potential to resist antibiotics currently used and might be difficult to manage in the future infections. Larger sample numbers generally have greater statistical powers. In practice, however, considering the time consuming and money spending, it could be extremely difficult to include a huge number of samples in a study. In this study, 30 samples were reported to indicate the statistical trends that larger clinical trials might exhibit.

\section{Bacteria identified in PVCs}

Bacterial species such as Staphylococcus spp., Streptococcus spp., and Pseudomonas spp. are well known as the major causes of colonization and related BSI [22,23], and they were also present on examined PVCs in this study. Nikkari et al. examined bacteria in venous blood from four healthy individuals [24]. They examined 192 clones and found Pseudomonas fluorescens (77\%), Acidovorax defluvii (17\%), Propionibacterium acnes (2\%), Staphylococcus epidermidis (2\%), Stenotrophomonas maltophilia (1\%) and Riemerella anatipestifer (1\%) [24]. The origins of these bacteria may be skin and/or blood [24]. The 
1 above study, despite some limitations, suggests the presence of bacterial communities in the

2 blood of healthy people. All bacterial species found in blood microbiota were also identified

3 in our study. These species are presumably cleared efficiently by the healthy reticulo-

4 endothelial system, but may be given the opportunity to colonize and persist on vascular

5 catheters when they are present. Specific conditions that then may lead to the establishment

6 of infection are yet to be determined.

7 The pyrosequencing library was dominated by Stenotrophomonas maltophilia, an

8 opportunistic pathogen that can cause serious infection and bacteraemia in critically ill

9 patients. The treatment of these can be difficult because Stenotrophomonas maltophilia is intrinsically resistant to many commonly used antimicrobial agents including most

11 cephalosporins, penicillins and quinolones [25-27]. Stenotrophomonas maltophilla has been

12 previously reported as the predominant species on arterial catheters [28].

13 It was noted that PVC samples with an increase in the proportion of Staphylococcus 14 (Firmicutes) showed a decrease of Stenotrophomonas (Gammaproteobacteria) at the same 15 time. A similar shift of bacterial composition was also seen in Propionibacterium 16 (Actinobacteria) and Stenotrophomonas (Gammaproteobacteria). These findings raise the 17 possibility that low level colonization of PVCs is the norm and changes in bacterial composition favouring the expansion of population of pathogens or opportunity pathogens such as Staphylococcus spp. might trigger the process of clinically significant bacterial colonization and BSI. Further investigation should be undertaken to determine whether these potential pathogens represent non-pathogenic variants or simply do not cause infection despite being present on PVC samples.

\section{Acknowledgements}


1 We thank Gillian Ray-Barruel for editorial assistance with the manuscript. LZ is supported by

2 National Health and Medical Research Council training clinical research fellowship,

3 Australian government (grant number: 597491). The research was supported by Griffith

4 Health Institute and Research Centre for Clinical and Community Practice Innovation project

5 grants from Griffith University, Australia.

\section{Conflict of Interest}

$7 \quad$ All authors declare no conflicts of interest relevant to this article.

8 
1. O'Grady NP, Alexander M, Burns LA, Dellinger EP, Garland J, Heard SO, Lipsett PA, Masur H, Mermel LA, Pearson ML, Raad, II, Randolph AG, Rupp ME, Saint S (2011) Guidelines for the Prevention of Intravascular Catheter-related Infections. Clin Infect Dis 52 (9):e162-e193. doi:cir257 [pii] 10.1093/cid/cir257

2. Ezingeard E, Coudrot M, Guyomarc'h S, Aubert G, Blanc JL, Bertrand JC, Tardy B, Zeni F (2009) Evaluation of colonisation of peripheral venous catheters inserted by prehospital emergency service teams (SMUR) in France. J Hosp Infect 72 (2):169-175. doi:DOI 10.1016/j.jhin.2009.01.007

3. Boyd S, Aggarwal I, Davey P, Logan M, Nathwani D (2011) Peripheral intravenous catheters: the road to quality improvement and safer patient care. J Hosp Infect 77 (1):37-41. doi:DOI 10.1016/j.jhin.2010.09.011

4. McHugh SM, Corrigan MA, Dimitrov BD, Cowman S, Tierney S, Hill ADK, Humphreys H (2011) Preventing infection in general surgery: improvements through education of surgeons by surgeons. $J$ Hosp Infect 78 (4):312-316. doi:10.1016/j.jhin.2011.03.023

5. Zhang L, Gowardman J, Rickard CM (2011) Impact of microbial attachment on intravascular catheter-related infections. Int J Antimicrob Agents 38 (1):9-15. doi:S0924-8579(11)00074-4 [pii] 10.1016/j.ijantimicag.2011.01.020

6. Widmer AF (2005) Infections and sepsis due to intvavascular catheters. Internist 46 (6):643-651 7. Mermel LA (2011) What Is The Predominant Source of Intravascular Catheter Infections? Clin Infect Dis 52 (2):211-212. doi:Doi 10.1093/Cid/Ciq108

8. Francolini I, Donelli G (2010) Prevention and control of biofilm-based medical-device-related infections. FEMS Immunol Med Microbiol 59 (3):227-238. doi:DOI 10.1111/j.1574695X.2010.00665.X

9. Jefferson KK (2004) What drives bacteria to produce a biofilm? FEMS Microbiol Lett 236 (2):163173

10. Donlan RM, Costerton JW (2002) Biofilms: Survival mechanisms of clinically relevant microorganisms. Clin Microbiol Rev 15 (2):167-193

11. Maki DG, Weise CE, Sarafin HW (1977) A semiquantitative culture method for identifying intravenous catheter-related infections. N Engl J Med 296:1305-1309

12. Bregenzer T, Conen D, Sakmann P, Widmer AF (1998) Is routine replacement of peripheral intravenous catheters necessary? Arch Intern Med 158 (2):151-156

13. Lee WL, Chen HL, Tsai TY, Lai IC, Chang WC, Huang CH, Fang CT (2009) Risk factors for peripheral intravenous catheter infection in hospitalized patients: a prospective study of 3165 patients. Am J Infect Control 37 (8):683-686. doi:10.1016/j.ajic.2009.02.009

14. Malach T, Jerassy Z, Rudensky B, Schlesinger Y, Broide E, Olsha O, Yinnon AM, Raveh D (2006) Prospective surveillance of phlebitis associated with peripheral intravenous catheters. Am J Infect Control 34 (5):308-312. doi:10.1016/j.ajic.2005.10.002

15. Caporaso JG, Kuczynski J, Stombaugh J, Bittinger K, Bushman FD, Costello EK, Fierer N, Pena AG, Goodrich JK, Gordon JI, Huttley GA, Kelley ST, Knights D, Koenig JE, Ley RE, Lozupone CA, McDonald D, Muegge BD, Pirrung M, Reeder J, Sevinsky JR, Turnbaugh PJ, Walters WA, Widmann J, Yatsunenko T, Zaneveld J, Knight R (2010) QIIME allows analysis of high-throughput community sequencing data. Nature methods 7 (5):335-336. doi:10.1038/nmeth.f.303

16. Dray S, Dufour AB (2007) The ade4 package: implementing the duality diagram for ecologists. J Statist Software $22(4): 1-20$

17. Rogers GB, Carroll MP, Bruce KD (2009) Studying bacterial infections through cultureindependent approaches. J Med Microbiol 58 (Pt 11):1401-1418. doi:jmm.0.013334-0 [pii] 10.1099/jmm.0.013334-0 
18. Mancini N, Carletti S, Ghidoli N, Cichero P, Burioni R, Clementi M (2010) The Era of Molecular and Other Non-Culture-Based Methods in Diagnosis of Sepsis. Clin Microbiol Rev 23 (1):235-+. doi:10.1128/Cmr.00043-09

19. Woo PCY, Lau SKP, Teng JLL, Tse H, Yuen KY (2008) Then and now: use of 16S rDNA gene sequencing for bacterial identification and discovery of novel bacteria in clinical microbiology laboratories. Clin Microbiol Infect 14 (10):908-934. doi:DOI 10.1111/j.1469-0691.2008.02070.x

20. Schabereiter-Gurtner C, Nehr M, Apfalter P, Makristathis A, Rotter ML, Hirschl AM (2008) Evaluation of a protocol for molecular broad-range diagnosis of culture-negative bacterial infections in clinical routine diagnosis. J Applied Microbiol 104 (4):1228-1237. doi:DOI 10.1111/j.13652672.2007.03648.x

21. Proctor RA, von Eiff C, Kahl BC, Becker K, McNamara P, Herrmann M, Peters G (2006) Small colony variants: a pathogenic form of bacteria that facilitates persistent and recurrent infections. Nature Reviews Microbiology 4 (4):295-305. doi:Doi 10.1038/Nrmicro1384

22. Van Donk P, Rickard CM, McGrail MR, Doolan G (2009) Routine Replacement versus Clinical Monitoring of Peripheral Intravenous Catheters in a Regional Hospital in the Home Program: A Randomized Controlled Trial. Infect Control Hosp Epidemiol 30 (9):915-917. doi:Doi 10.1086/599776

23. Bouza E, Burillo A, Munoz P (2002) Catheter-related infections: diagnosis and intravascular treatment. Clin Microbiol Infect 8 (5):265-274

24. Nikkari S, McLaughlin IJ, Bi WL, Dodge DE, Relman DA (2001) Does blood of healthy subjects contain bacterial ribosomal DNA? J Clin Microbiol 39 (5):1956-1959

25. Wang WS, Liu CP, Lee CM, Huang FY (2005) Stenotrophomonas maltophilia bacteremia in adults: four years' experience in a medical center in northern Taiwan. . J Microbiol Immunol Infect 37:359365

26. Friedman ND, Korman TM, Fairley CK, Franklin JC, Spelman DW (2002) Bacteraemia due to Stenotrophomonas maltophilia: An analysis of 45 episodes. J Infect 45 (1):47-53

27. Ryan RP, Monchy S, Cardinale M, Taghavi S, Crossman L, Avison MB, Berg G, van der Lelie D, Dow JM (2009) The versatility and adaptation of bacteria from the genus Stenotrophomonas. Nature reviews Microbiology 7 (7):514-525

28. Zhang L, Sriprakash KS, McMillan D, Gowardman JR, Patel B, Rickard CM (2010) Microbiological pattern of arterial catheters in the intensive care unit. BMC Microbiol 10:266-275 


\section{Figure legends}

2 Fig. 1. Microbial phyla detected in (a) PVC samples, and (b) outer surfaces and inner 3 surfaces of PVCs. A represent inner surface of PVCs and B represents outer surfaces of $4 \quad$ PVCs. Each column represents one sample.

5 Fig. 2. Heatmap show the relative abundance of bacterial genera across PVC samples of 615 patients. A represents inner surface of PVCs and B represents outer surfaces of PVCs.

7 Each column represents one sample.

8 Fig. 3. The PCoA plot of bacterial communities on individual PVC sample as 9 determined using UniFrac. AN represents PVC samples from patients with antibiotic treatment. NOAN represents PVC samples from patients without antibiotic treatment.

11 Fig. 4. Monte Carlo permutation analysis of bacterial communities on PVCs from patients with 12 and without antibiotic treatment. Red ball represents with antibiotic treatment and green ball 13 represents no antibiotic treatment.

14 Fig. 5. OTUs are significantly different on PVCs from patients with and without 15 antibiotic treatment $(\mathbf{P}<\mathbf{0 . 0 5})$. AN represents with antibiotic treatment and NOAN 16 represents no antibiotic treatment. 
Fig. 1 a

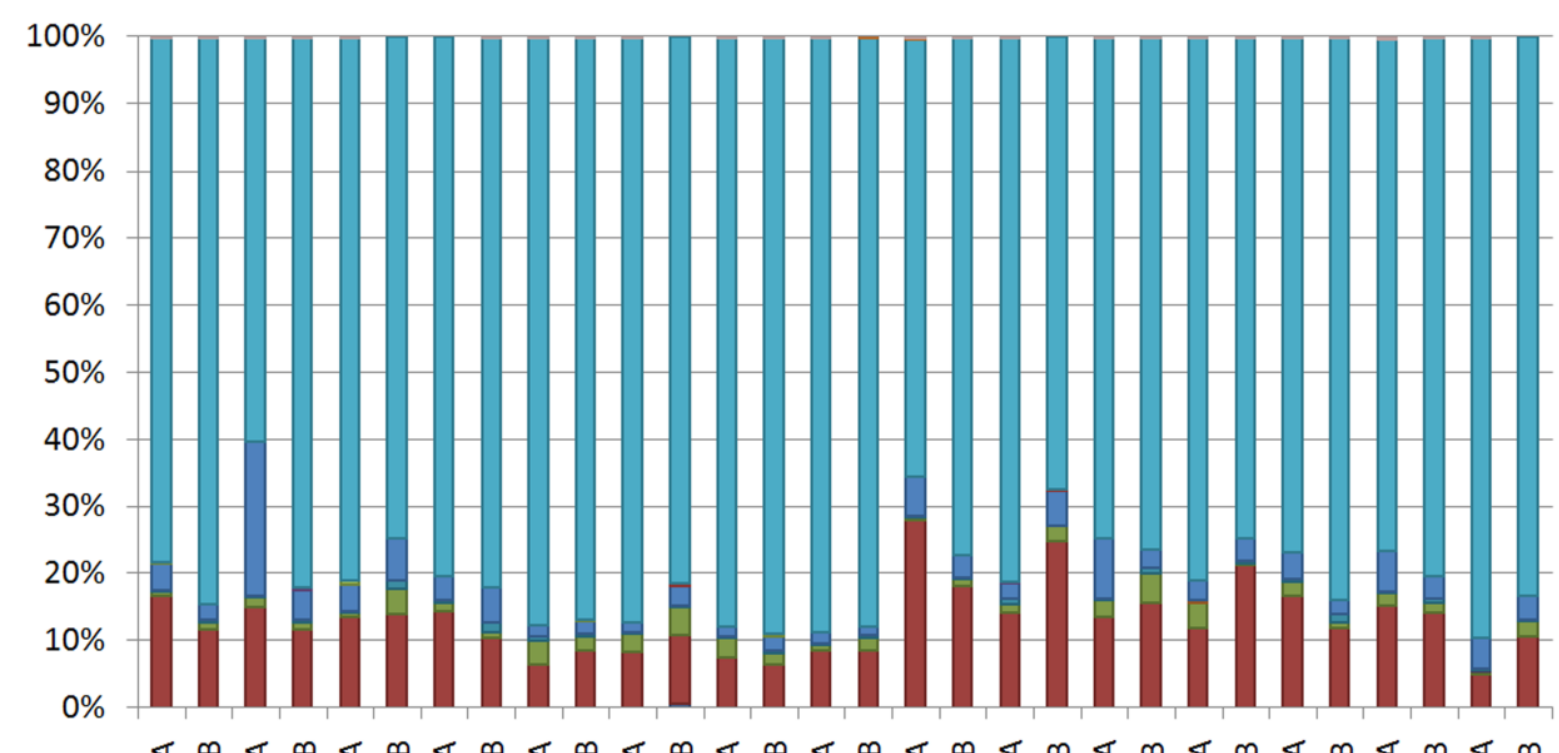

ฐ

Acidobacteria

Cyanobacteria

Gemmatimonadetes

Thermotogae

\section{Actinobacteria} Deinococcus-Thermus

Planctomycetes

Bacteroidetes

- Firmicutes

Proteobacteria
Chloroflexi

Fusobacteria

$\square$ TM7 
Fig. $1 b$

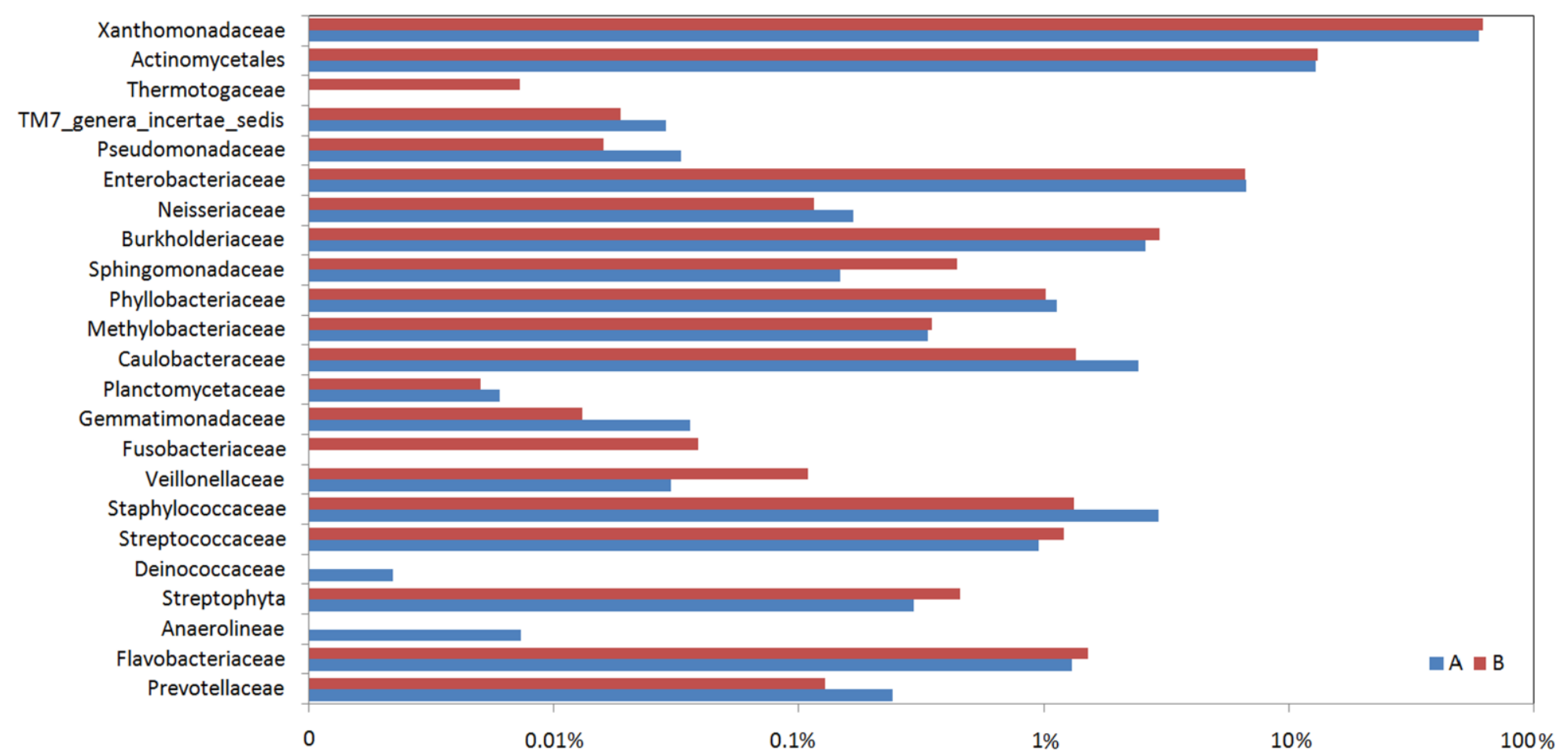


Fig. 2.
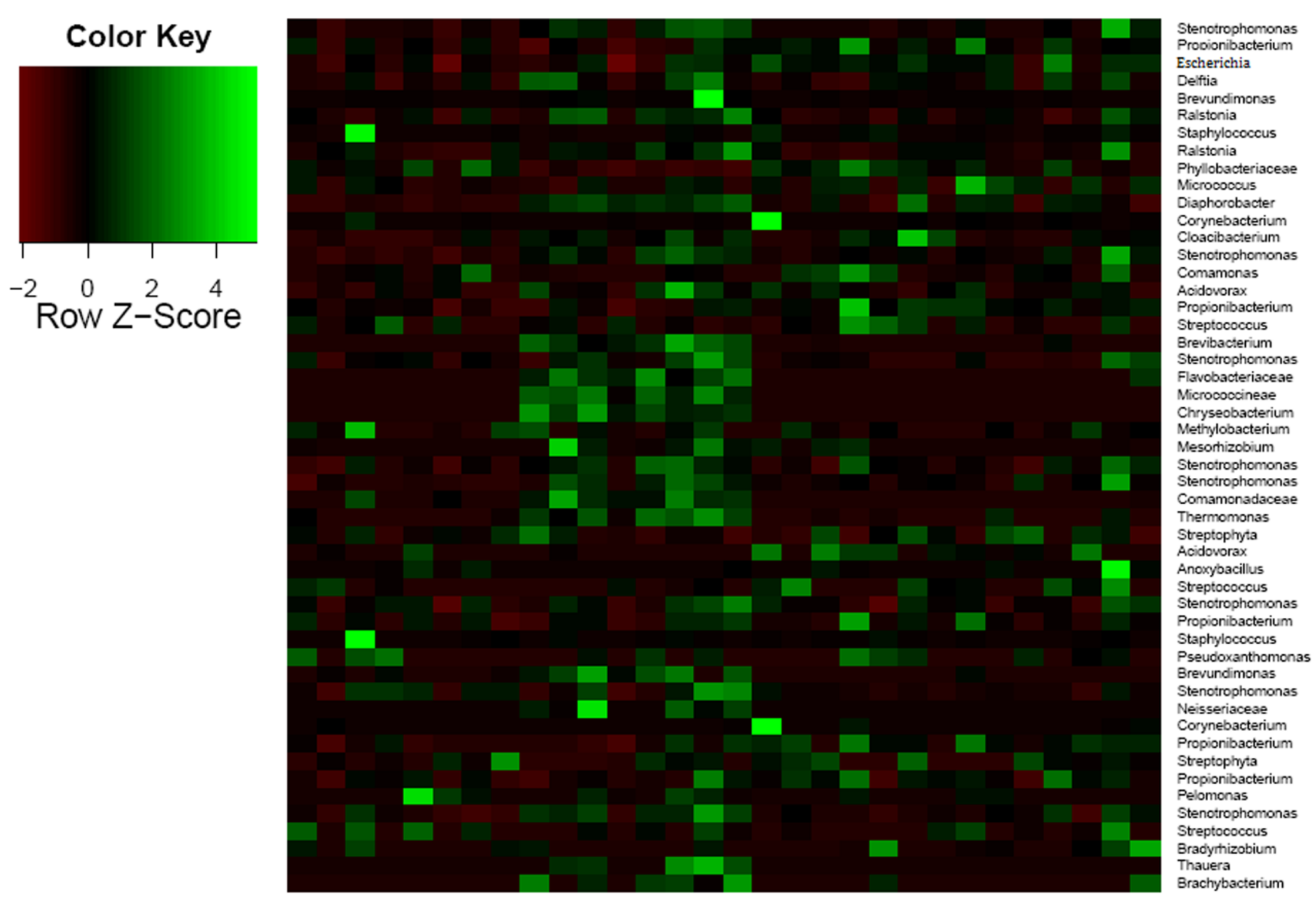

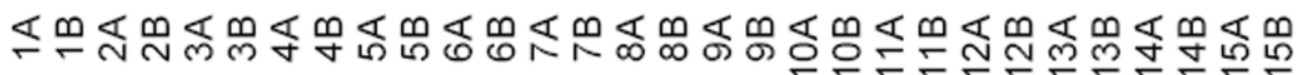


Fig. 3 .

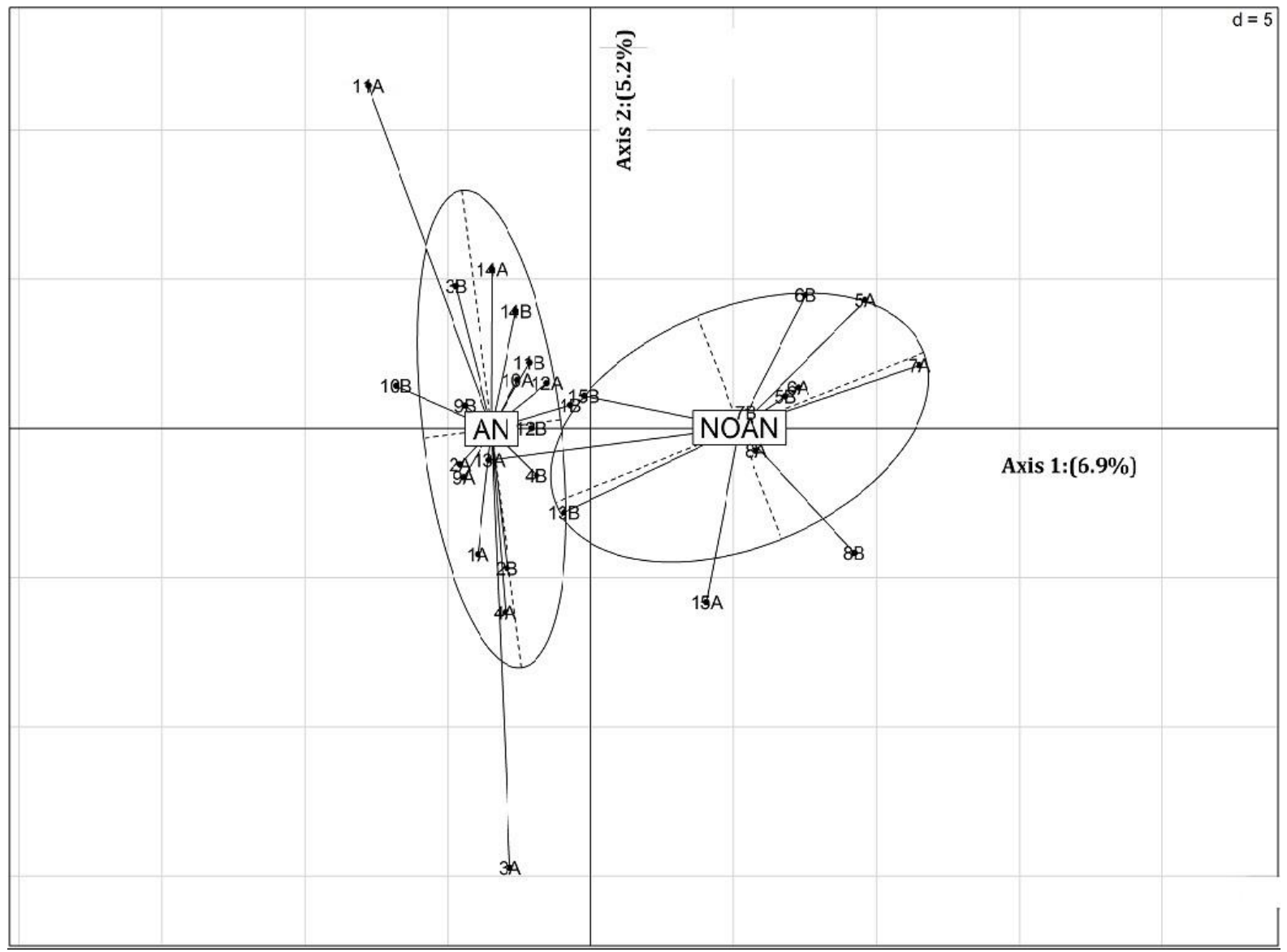


Fig. 4.

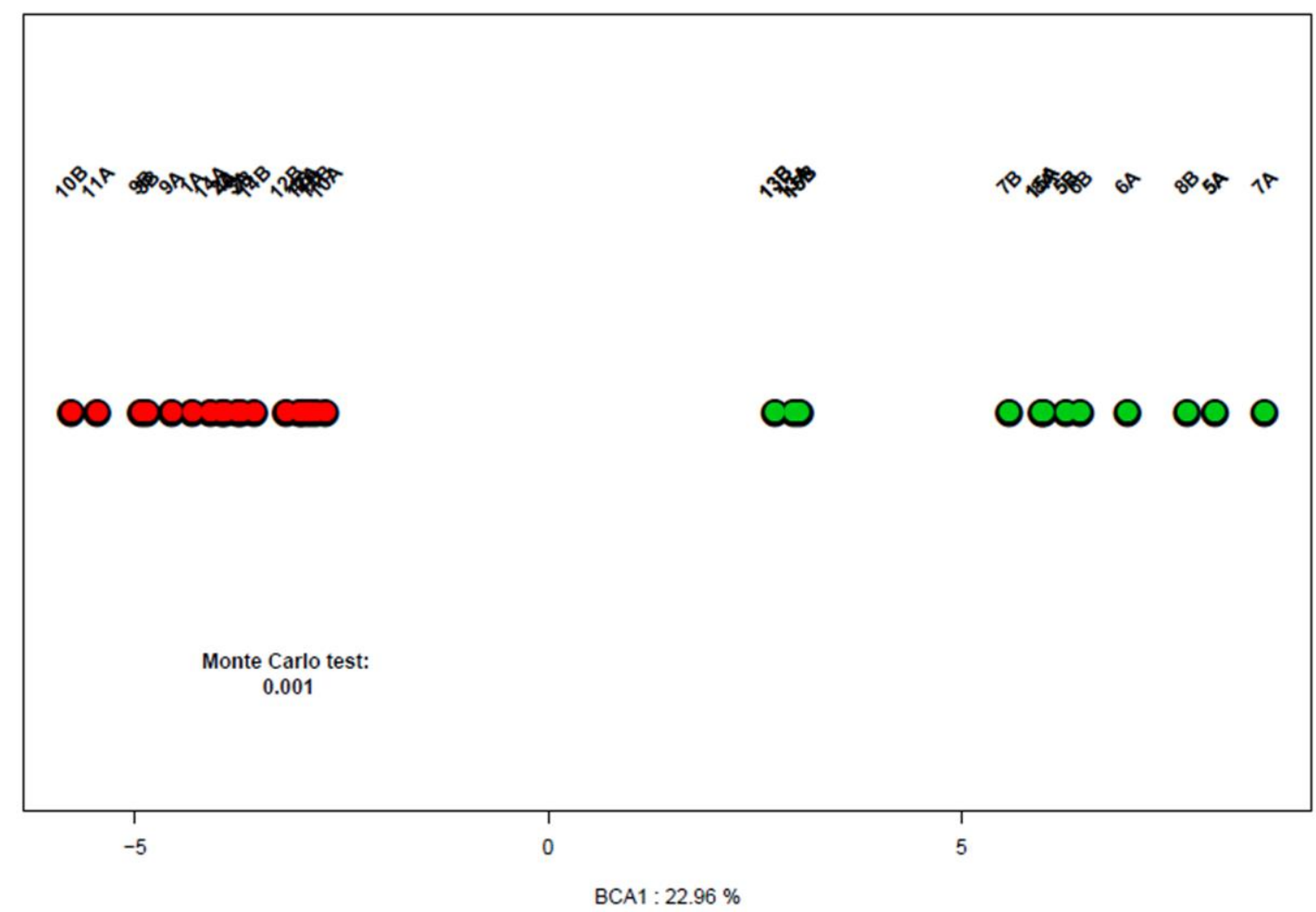


Fig. 5.
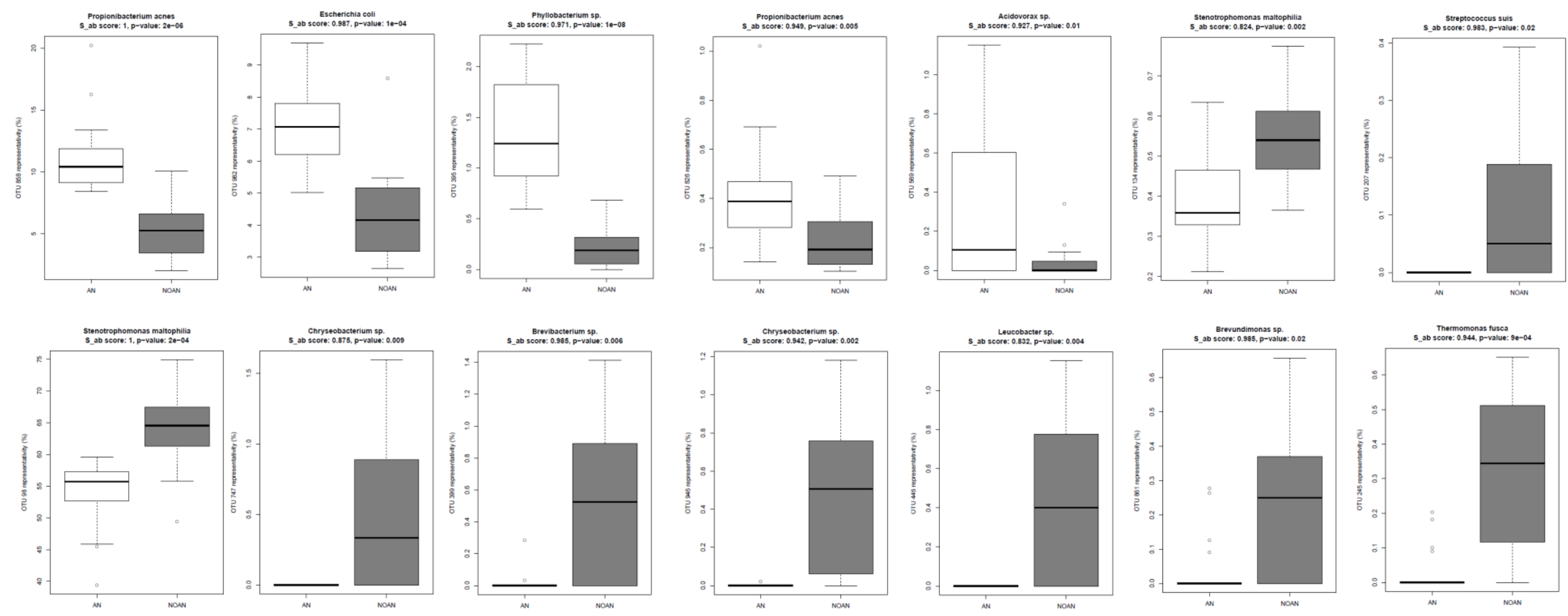